\title{
¿Educación inclusiva en la Enseñanza Superior?: el caso del alumnado con discapacidad
}

\author{
Anabel Moriña Díez ${ }^{1}$ \\ Víctor Hugo Perera Rodríguez ${ }^{2}$
}

\section{Introducción}

El número de alumnado universitario con discapacidad está incrementándose año tras año en un número importante de países (HADJIKAKOU; HARTAS, 2008; HOLLOWAY, 2001; KONUR, 2002, 2006). A pesar de este hecho, no es menos cierto que este grupo de estudiantes sigue siendo minoritario y aún se necesitan experiencias inclusivas en la Enseñanza Superior (ES) que den cuenta de cómo se está avanzando en este sentido.

Algunas de las cuestiones que ayudan a explicar este cambio en las estadísticas tienen que ver con el desarrollo de una legislación nacional que reconoce ese derecho ${ }^{3}$ (FULLER et al., 2005; RIDDELL et al., 2007), la implementación

1 US - Universidad de Sevilla. Departamento de Didáctica y Organización Escolar. Sevilla - Sevilla - Andalucía - España. anabelm@us.es

2 US - Universidad de Sevilla. Departamento de Didáctica y Organización Escolar. Sevilla - Sevilla - Andalucía - España.vhperera@us.es

3 En el caso de Espańa, los estudiantes con discapacidad están presentes en la Ley Orgánica 4/2007 de Universidades. A este respecto se establece que se debe garantizar el principio de igualdad de oportunidades y la no discriminación, además de la garantía de la obligatoriedad de que los entornos universitarios sean accesibles - edificios, instalaciones y dependencias. Más en concreto, en la Universidad de Sevilla, institución en la que se desarrolla este estudio, se aprobó una normativa para la atención 
de prácticas educativas inclusivas, la incorporación de las Nuevas Tecnologías, la creación de servicios en las universidades para apoyar al alumnado con discapacidad, etc. De hecho, en la década pasada muchos países desarrollados han puesto en marcha acciones para hacer que las universidades sean más accesibles para las personas con discapacidad, llegando a estar progresivamente más comprometidas con los procesos de inclusión (BARNES, 2007; JACKLIN et al., 2007). Desde este punto de vista, Tinklin, Riddell y Wilson (2004) expresan que los estudiantes con discapacidad suponen un reto para la Universidad, no sólo en términos de eliminar las barreras arquitectónicas, sino también en relación a un acceso mucho más amplio al currículo, enseñanza, aprendizaje y evaluación. Asimismo, Fuller et al., (2005), Fuller, Bradley y Healey (2004), Hurst (1996), Moswela y Mukhopadhyay (2011) y Wehman (2006) afirman que la Universidad debe ser considerada como un vehículo para mejorar la calidad de vida de las personas con discapacidad. Es más, desde la década de 1990 se viene insistiendo en la necesidad de que el aprendizaje sea inclusivo en la Enseñanza Superior (TOMLINSON, 1996). Sin embargo, paralelamente se ha concluido que este sistema se encuentra entre las instituciones más excluyentes para su acceso y permanencia (BAUSELA HERRERAS, 2002; DÍAZ SÁNCHEZ, 2000; KONUR, 2006; MORENO, 2005; ONOFRE, 2006).

En este sentido, la educación inclusiva en la Universidad ha ido avanzando como un movimiento que reta cualquier situación de exclusión. Cada vez son más las voces que reclaman un contexto de enseñanza y aprendizaje en la Enseñanza Superior en el que todos los estudiantes puedan aprender, participar y pertenecer (BARNES, 2007; FULLER; BRADLEY; HEALEY, 2004; FULLER et al., 2004; HURST; 1996; JACKLIN et al., 2007; MORIŃA; CORTÉS; MELERO, 2014; MOSWELA; MUKHOPADHYAY, 2011).

Sin embargo, como muestran los resultados de investigaciones realizadas en distintos contextos internacionales sobre las experiencias de personas con discapacidad en el sistema universitario, las vivencias del alumnado no siempre han sido positivas y las universidades se encuentran entre las instituciones más excluyentes para el acceso y permanencia del alumnado con discapacidad (BORLAND; JAMES, 1999; BOXALL; CARSON; DOCHERTY, 2004; CLAIBORNE et al., 2010; FOREMAN et al., 2001; FULLER; BRADLEY; HEALY, 2004; HOLOWAY, 2001; HOPKINS, 2011; JACKLIN et al., 2007; MOSWELA; MUKHOPADHYAY, 2011; NIELSEN, 2001; OLNEY; BROCKELMAN, 2003; PROWSE, 2009; RIDDELL; WILSON; TINKLIN, 2002; RYAN; STRUHS, 2004; SHEVLIN; KENNY; MCNEELA, 2004; TINKLIN; HALL, 1999; TINKLIN; RIDDELL; WILSON, 2004). Como explican estos trabajos, aunque existe una legislación

académica de este colectivo, incluyendo en ésta la necesidad de realizar adaptaciones curriculares por parte del profesorado y la gratuidad o coste cero en la primera y posteriores matrículas universitarias (Acuerdo 8/CG 9-12-08, BOUS 12 enero 2009). 
anti-discriminación, en la práctica este alumnado se encuentra con significativas barreras que obstaculizan sus procesos de participación y aprendizaje.

Por otro lado, aunque es cierto que la Enseńanza Superior puede suponer una oportunidad para que los estudiantes puedan participar plenamente en la sociedad, también los es que un número importante de investigaciones ha identificado barreras significativas que restringen la participación, el progreso y el éxito en la Universidad de los estudiantes con discapacidad. Esas barreras son principalmente actitudinales, estructurales y referidas a recursos (MOSWELA; MUKHOPADHYAY, 2011). La participación en los entornos educativos en la Enseñanza Superior a veces está restringida por currículos inaccesibles, actitudes negativas del personal y barreras arquitectónicas (FERNI; HENNING, 2006; OLIVER; BARNES, 2010). Además, Adams y Holland (2006a, 2006b) explican que los estudiantes con discapacidad se enfrentan a barreras adicionales y retos que se hacen visibles cuando ellos se esfuerzan por continuar su educación. Desde el modelo social de discapacidad se entiende que es precisamente la sociedad, y en el caso de este estudio, la universidad, quién genera las barreras que obstaculiza la inclusión de los universitarios con discapacidad (OLIVER, 1990).

De acuerdo con este modelo, las universidades deberían evitar el uso de etiquetas médicas para identificar las necesidades de aprendizaje del alumnado con discapacidad, y debería hacer esfuerzos para instituirlo como parte de una práctica diaria con estrategias de enseñanza inclusivas (MATTHEWS, 2009). En este sentido, las prácticas de enseñanza y aprendizaje pueden suponer una barrera para el aprendizaje del alumnado con discapacidad cuando llegan a la Universidad (FULLER; BRADLEY; HEALEY, 2004; POWELL, 2003; TINKLIN; RIDDELL; WILSON, 2004). En síntesis, el modelo médico ve la discapacidad como un "problema individual" (ARMSTRONG; BARTON, 1999). En contraste, desde el modelo social, la discapacidad no es una tragedia personal, una anormalidad o una necesidad de curar la enfermedad. Es una forma de discriminación y opresión (BARTON, 1998). Desde esta perspectiva, se plantea la necesidad de reestructurar los entornos educativos de tal manera que todos los estudiantes puedan participar (OLIVER, 1990).

Por otro lado, los conceptos de ayuda y barrera, en este trabajo, quedan vinculados con el modelo social de discapacidad (BARON; PHILLIPS; STALKER, 1996; FULLER et al., 2005; TINKLIN; HALL, 1999). En este sentido, entendemos las ayudas como elementos del contexto educativo que contribuyen a que el alumnado esté incluido social y educativamente en las aulas (actitudes positivas, metodologías adecuadas, relaciones sociales, etc.). Las barreras, por el contrario, son reconocidas como obstáculos a la inclusión que dificultan o limitan el aprendizaje, pertenencia y participación activa, en condiciones de igualdad, en los procesos educativos. Por ejemplo, tal y como explica Messiou (2006), una persona puede experimentar exclusión cuando no puede acceder al currículo, no 
se le da las oportunidades para participar en las clases, sus capacidades no son valoradas, es rechazada por sus iguales o incluso cuando se le niega el derecho a las relaciones sociales. O como también han descrito Ainscow y Miles (2009) el concepto de "barreras" hace referencia a cómo, por ejemplo, la falta de recursos o de experiencia o la existencia de un programa, de métodos de enseńanza y de actitudes inadecuadas pueden limitar la presencia, la participación y el aprendizaje de determinados estudiantes. De hecho, se ha reconocido que aquellos estudiantes, entre los que se encuentra el alumnado con discapacidad, que experimentan estas barreras, pueden ser considerados "voces escondidas".

En investigaciones previas, en diversas etapas educativas, ya se ha explorado las perspectivas y experiencias de estudiantes (BEAUCHAMP-PRYOR, 2012; HOPKINS, 2011, NORWICH; KELLY, 2004; RILEY; DOCKING, 2004; RUDDUCK, 1996; STEVENS, 2009). En otros trabajos se ha argumentado que escuchar a personas vulnerables a procesos de exclusión podría dar información relevante con relación a la educación inclusiva (BIKLEN, 2000; CORBETT; SLEE, 2000; MCCLUSKEY, 2008; MUNN; LLOYS, 2005; TANGEN, 2008). Por lo tanto, en estas publicaciones se concluye que las voces de personas que experimentan procesos de exclusión deberían tener un rol central en el proceso de inclusión ya que puede iluminar el camino a seguir.

En este artículo, tomando como referente las narraciones de los estudiantes que han participado en esta investigación es posible reflexionar acerca de cómo las diversas actitudes, prácticas y conductas experimentadas en determinadas situaciones en la universidad, influyen en sus trayectorias académicas. Desde estas voces, asimismo, se formulan una serie de propuestas para caminar hacia la inclusión en la ES.

\section{Diseño metodológico de la investigación}

Los resultados que presentamos en este artículo pertenecen a una investigación más amplia financiada por el Ministerio de Ciencia e Innovación de España: "Barreras y ayudas que los estudiantes con discapacidad identifican en la Universidad". Este estudio que tiene una duración de 4 años (2011-2014) está llevándose a cabo por un equipo de investigación compuesto por profesorado de la Universidad de Sevilla perteneciente a diferentes áreas y campos de conocimiento (Ciencias de la Educación, Ciencias Económicas, Ciencias de la Salud y Ciencias Experimentales). El propósito del mismo es estudiar, desde la perspectiva de los estudiantes universitarios con discapacidad, las barreras y ayudas que identifican en su acceso, trayectoria y resultados en la Universidad. En concreto, con esta investigación tratamos de responder a tres objetivos: 1) Identificar, describir y explicar las barreras y las ayudas que los estudiantes con discapacidad perciben en su acceso, trayectoria y resultados en la Universidad como Institución; 2) Identificar, describir y explicar las barreras y ayudas que los estudiantes con discapacidad 
experimentan en las aulas universitarias; y 3) Diseñar una guía de formación online para el profesorado universitario con el fin de dar respuesta a las necesidades educativas del alumnado con discapacidad.

Para dar respuestas a estos objetivos, a través de una metodología biográfico-narrativa, nos hemos planteado distintas fases de investigación. En la primera fase contemplamos dos momentos. En el primero, se han realizado diversos grupos de discusión (al menos un grupo de discusión para cada uno de los cinco campos de conocimiento) ${ }^{4}$, y entrevistas individuales (orales y escritas). En este primer momento han participado un total de 44 alumnos. En un segundo momento se han realizado micro-historias de vida con 16 estudiantes que participaron en el momento anterior. Estas historias se caracterizan por ser temáticas, ya que nos hemos centrado en un período particular de sus vidas, la trayectoria universitaria. Para su elaboración, los instrumentos de recogidas de datos que se han utilizando son tres: líneas de vida, entrevistas focalizadas y auto-informes. En la segunda fase de investigación, aún en desarrollo, se han retomado las historias de vida iniciadas con sólo 8 de los estudiantes que participaron en el segundo momento y se están realizando lo que se conoce en metodología biográfico-narrativa como historias de vida en profundidad y polifonía de voces (FRANK, 2011). Para la realización de estas historias se ha recurrido a técnicas de recogida de datos como entrevista en profundidad, fotografía, entrevistas a otras personas clave en la vida de cada estudiante y observaciones. Como última fase del estudio se pretende diseñar una propuesta de formación online para que el profesorado pueda dar respuesta a la diversidad.

En concreto, este artículo, que se centra íntegramente en el objetivo 1 de esta investigación y en las fases I y II, ha tomado como población del estudio los estudiantes con discapacidad matriculados en la Universidad de Sevilla (US) en el curso 2009/2010. Sobre esta Universidad podemos decir que en ese momento contaba con un total de 445 estudiantes con discapacidad (0.6\%) frente a los 72.358 estudiantes con los que cuenta la Institución.

Sobre el perfil de la muestra podemos comentar, en líneas generales, que las edades de estas personas oscilan entre los 19 y los 59 ańos, siendo la media de edad 30,5. De entre estas personas, 22 son hombres y otros 22 son mujeres. Tomando en consideración el conjunto total de estudiantes, un $25 \%$ está en primero de carrera, un $16 \%$ en segundo, un $25 \%$ en tercero, un $14 \%$ en cuarto y un $9 \%$ en quinto. El resto, aproximadamente un $11 \%$, realizan estudios de posgrado, en másteres oficiales. El 63\% de los estudiantes han permanecido de uno a cinco ańos en la

4 Los campos de conocimiento que se contemplan en la Universidad de Sevilla son: Ciencias de la Salud; Ciencias Experimentales; Ciencias Sociales y Jurídicas; Ingeniería y Tecnología; Humanidades. 
universidad, mientras que el 37\% de los estudiantes restantes han estado más de cinco años. Es importante destacar que un $14 \%$ de los estudiantes, tomados del dato anterior, han estado diez o más años en la universidad. Por último, en cuanto al tipo de discapacidad de este alumnado, de acuerdo a la denominación usada por esta Universidad, el 38\% es alumnado con discapacidad física, el 15\% discapacidad psíquica, el $36 \%$ discapacidad sensorial y el $11 \%$ presenta dificultades asociadas a algún problema orgánico (asma, enfermedades degenerativas, etc.).

Finalmente, en el tratamiento de los datos hemos realizado un doble análisis. Para la elaboración de cada historia de vida se ha llevado a cabo lo que en la literatura se denomina análisis narrativo en el sentido que proponen Goodley et al. (2004). Por otro lado, para el análisis comparativo de la información recogida con todas las técnicas y personas participantes se ha realizado un análisis estructural (RIESSMAN, 2008), siguiendo a Miles y Huberman (1994), y utilizando un sistema inductivo de categorías y códigos incluido en el programa de análisis de datos MaxQDA10.

\section{Resultados}

En este apartado del artículo se presentan los resultados referidos a las barreras y ayudas que los estudiantes con discapacidad identifican en la Universidad de Sevilla, específicamente aquellos que hacen referencia a la Universidad como Institución y al Servicio de Atención a la Comunidad Universitaria (SACU) ${ }^{5}$, y más concretamente a la Unidad de Atención a Estudiantes con Discapacidad $(\mathrm{SAD})^{6}$. En estos temas, se explorarán los elementos que actúan como obstáculos y facilitadores, ofreciendo puntos de reflexión que permitan analizar los procesos de inclusión educativa en la Universidad de Sevilla.

\section{¿Alzando muros o tendiendo puentes en la arquitectura universitaria?}

La Universidad de Sevilla se describe como una institución en la que perviven barreras y ayudas en la vida académica de los estudiantes que participan en esta investigación. Son muchos los aspectos organizativos, docentes y arquitectónicos que concurren de forma reiterada, comprometiendo la actividad académica de los estudiantes con discapacidad.

5 Servicio universitario que integra varias unidades y programas de asesoría, atención y apoyo a la comunidad universitaria en ámbitos como la salud, empleo, apoyo jurídico y pedagógico, entre otros.

6 Unidad adscrita al Servicio de Asistencia a la Comunidad Universitaria que tiene el compromiso institucional de conseguir la inclusión plena y efectiva en el ámbito universitario de todos aquellos miembros de la Comunidad Universitaria que presenten algún tipo de discapacidad. 
Uno de los problemas que se identifican es el desconocimiento por parte de la Comunidad Universitaria, incluyendo al profesorado, de la normativa que regula la atención académica a los estudiantes con discapacidad y las subsecuentes trabas organizativas y actitudinales que deriva de esto. La universidad de Sevilla cuenta desde el curso 2008/2009 con una normativa que avala, resguarda y protege los derechos de los estudiantes, por lo que su difusión efectiva es necesaria para que pueda cumplirse. Aún con esto, la principal consecuencia de su desconocimiento afecta a la organización interna y al quehacer diario de cada Facultad, ya que muchas de las respuestas y tomas de decisiones no tienen en cuenta dicha normativa.

$\mathrm{El}$ acceso a la universidad también supone para estos estudiantes un momento complejo ocasionado por la desinformación. Esto genera un sentimiento generalizado de desconcierto en los estudiantes al no disponer de orientaciones que les facilite conocer la institución y su funcionamiento, una vez que acceden a la universidad.

Una de las barreras más comúnmente identificada es el exceso de burocracia que el alumnado se encuentra en el momento de solicitar, en convocatorias oficiales, ayudas relativas a sus necesidades. La solicitud de las ayudas se describe como un procedimiento formal que ralentiza la obtención de las mismas, llegando en ocasiones una vez que finalizan los estudios. Esto ocurre con las ayudas económicas y las de movilidad para realizar estancias en el extranjero, a excepción de las becas que se reciben para las deducciones y pago de las matrículas, que son de aplicación inmediata, señalándose en esta investigación como una de las principales ayudas. La gestión administrativa socava la paciencia de los estudiantes hasta el punto de acabar presentándose sin cita ni preaviso en los despachos de los responsables para realizar tal petición.

En el otro lado de la balanza, a nivel institucional, una de las ayudas mejor valoradas por los participantes del estudio está relacionada con la inmediatez y continuidad en la información que reciben sobre las ayudas, becas, ofertas de trabajo y cursos de formación. La universidad se sirve de una web para alojar y publicar este tipo de información actualizada. No obstante, hay quienes reclaman la existencia de algún organismo, departamento o figura de apoyo que los ayuden durante su primer ańo académico, ya que afirman que se sienten desamparados y dependientes de la buena voluntad del personal de la universidad.

La idea anterior se apoya en las aportaciones de estudiantes que informan que en otras universidades existe una figura de apoyo e incluso software informático que les indica, a través de la realización de la matrícula, con qué persona u organismo ha de ponerse en contacto el alumnado con discapacidad para su orientación e inserción en la universidad. En base a esto, la mayoría de los participantes coinciden en que la universidad no facilita información a los profesores sobre su discapacidad por lo que se considera igualmente un obstáculo en su contra, ya que éstos no pueden planificar la programación de las clases con suficiente antelación. Precisamente, 
esta información es recogida durante el periodo de matriculación, indicándose si se tiene o no discapacidad, el tipo y grado, y aseguran que dicha información no llega al profesorado. De igual modo es preocupante que no se sepa exactamente a dónde va a parar y qué utilidad se les da a esta información. Aseguran que si esta información llegara a conocimiento del profesorado y, en consecuencia, conocieran de antemano sus necesidades educativas, probablemente tendrían sus asignaturas adaptadas, solventándose muchos de los problemas que de esto pudiera derivarse como es el caso de mantener mejor el ritmo de las clases.

En cuanto a las ayudas que estos estudiantes con discapacidad puedan necesitar por parte de la universidad como institución, se entiende que depende de la buena voluntad del personal de administración y servicio, del profesorado y del alumnado. Denuncian que dichas ayudas no deben derivar exclusivamente de la buena voluntad sino del derecho y el deber que rija una normativa y que oriente la actuación de la Comunidad Universitaria. No obstante, perciben esto como algo positivo ya que supone un cambio de mentalidad y de sensibilidad ante las personas con discapacidad.

En relación al profesorado se aprecia el compromiso de atender bien a estos estudiantes. Se valora el hecho de que sean flexibles con el ritmo de aprendizaje, que faciliten la entrega de los trabajos y el material de estudio a través de la plataforma virtual de aprendizaje de la universidad, así como que compartan con anticipación las presentaciones de cada tema para que puedan verlas en su propio portátil, etc. Hay, sin embargo, una percepción general por parte de los estudiantes que cuestiona la formación del profesorado para atender al alumnado con discapacidad. Una de las consecuencias de esta necesidad de formación del profesorado es que se encuentran desprovistos de recursos que les ayude a cubrir las demandas académicas de estos estudiantes y esto se refleja en que las asignaturas no se adaptan, pese a existir una normativa que lo indique.

Igual importancia se le otorga a la creación de un servicio interno que pueda transcribir los apuntes para el alumnado que tenga una discapacidad visual, o que realice programas especiales, en atención al trabajo que vienen haciendo organismos externos como es el caso de la ONCE. 7 Por ello, los estudiantes creen que el profesorado debe dejar sus materiales por adelantado para que pueda adaptarse a tiempo, y no dejarlos paulatinamente durante el curso, pues en el caso de los estudiantes con discapacidad visual, les dificulta llevar el ritmo normal de las clases.

En relación a las asignaturas, encuentran ciertas ayudas cuando se trata de cambiar el turno en los horarios para poder recibir la materia, el acceso a la plataforma virtual de aprendizaje o la propia flexibilidad en la convocatoria de

7 La Organización Nacional de Ciegos Españoles (ONCE) es una Corporación sin ánimo de lucro con la misión de mejorar la calidad de vida de las personas ciegas y con discapacidad visual de toda España. 
exámenes. No obstante, el alumnado experimenta que el Plan de Bolonia todavía no se encuentra integrado en su totalidad en la organización y planificación de las asignaturas, y explican que las asignaturas que anteriormente eran anuales ahora son cuatrimestrales, que se han reducido en tiempo y duración pero no en materia, por lo que se encuentran saturados de trabajo y material de estudio. De igual modo, entienden que en determinadas carreras las asignaturas no debieran ser de obligada elección sino de libre opción y de acuerdo a sus horarios y necesidades.

Respecto a las ayudas otorgadas a los estudiantes dependiendo de la discapacidad, son varias las diferencias estudiadas en cuanto al destino de las mismas. Por lo general, aseguran que las ayudas ofrecidas son para personas con discapacidad física o sensorial pero no hay para personas con discapacidad psíquica u orgánica. Este hecho se agrava aún más cuando la información y difusión sobre dichas ayudas no llega a este colectivo de estudiantes.

Por último, respecto a la arquitectura e infraestructura, se perciben mejoras sustanciales en los espacios (por ejemplo, la anchura de los pasillos y entradas al aula) y en los edificios (donde se evidencian reparaciones, arreglos y adaptaciones). Sin embargo, en la organización del espacio físico hay algunas clases que se encuentran masificadas, impidiendo la puesta en práctica de determinadas metodologías que promueven el trabajo en grupo y la participación mediante debates, dificultando el acceso a determinadas aulas, afectando a su organización, motivos por los que abandonan algunas asignaturas. Esta situación genera cierta indignación, pues la propia normativa establece que los espacios deben ser accesibles e igualitarios para todos. La accesibilidad a determinadas aulas se ve quebrantada al no estar adaptado el mobiliario a sus necesidades (por ejemplo, mesas y sillas atornilladas). La experiencia del alumnado asegura que la estructura de las aulas no facilita el proceso de aprendizaje y que los materiales que se utilizan en las mismas para el desarrollo de las asignaturas, como es el caso de los retroproyectores, pizarras, tarima etc., no son accesibles al alumnado con determinada discapacidad.

\section{¿Abriendo o cerrando puertas desde el servicio de atención al alumnado con discapacidad?}

En referencia al SACU, y más concretamente al SAD, los participantes del estudio se mostraron satisfechos con la labor que realizan; así como con el personal encargado de atender las necesidades del alumnado con discapacidad. En la medida de sus posibilidades, se comprende que este servicio resuelve con eficacia los problemas y ayuda a cubrir las necesidades de estos estudiantes.

En el ámbito universitario, se percibe que la inclusión es menos difícil gracias a las ayudas recibidas por este Servicio. Además, se valora la excelente labor realizada por la persona encargada del SAD. No obstante, hay una manifiesta 
insatisfacción por la escasa cobertura que da el Servicio, ya que una sola persona es la que debe atender a todos los estudiantes con discapacidad en la Universidad de Sevilla. Se considera, por tanto, que son escasos los medios que garantizan una atención de calidad a toda la comunidad universitaria con discapacidad.

Además, es preciso visibilizar que la US cuenta actualmente con 26 centros docentes integrados en la propia ciudad, desplegándose en varias zonas urbanas (Ramón y Cajal/Pirotecnia, Macarena, Reina Mercedes y Cartuja). En este modelo de campus universitario, como comentan los universitarios, la ubicación geográfica del SAD queda aislada puesto que su ubicación está centralizada en una única sede. En consecuencia, se reclama nuevas sedes por Facultad o por zona, que eviten que determinados estudiantes, por causas distintas, no puedan visitarla según les convengan.

De igual modo, la movilidad y accesibilidad del edificio donde se encuentra la sede del SACU son dos aspectos que se reafirman como obstáculos que deben superarse. También se considera una barrera la falta de información sobre la labor que realiza el SACU, los servicios que ofrecen, las condiciones de las becas e incluso de su existencia. Antes incluso, les llega más información a través de otros organismos de mayor envergadura.

Entre las ayudas que el SACU proporciona, la más valorada, y a su vez criticada, es la del estudiante colaborador. ${ }^{8}$ La función del alumno colaborador consiste en facilitar el acceso a la información al alumnado con discapacidad, el apoyo en los desplazamientos y la toma de apuntes en clase, encontrándose en muchas ocasiones con problemas para la comprensión de los mismos. Estas personas aceptan las becas motivadas por la obtención de créditos que luego son reconocidos en la secretaría de su centro y por la ayuda económica con la que el SACU les gratifica. Es una ayuda que los estudiantes discapacitados tildan de utópica ya que no tienen efecto ni beneficio real en sus estudios.

Una última barrera se centra en la rígida burocratización del SACU respecto a las ayudas que pueden conceder. Muchos estudiantes con discapacidad que acceden al primer curso no tienen constancia de la existencia de este Servicio. Piensan que pueden perder oportunidades para beneficiarse de las ayudas, pues se suele dar el caso en el que cuando se recibe la información el plazo de solicitud de las ayudas ha finalizado.

8 El programa estudiante colaborador es una de las iniciativas que integra el Proyecto Contigo, cuyo objetivo es crear un grupo de personas comprometidas que participen de forma activa en el despliegue de acciones solidarias en relación a la discapacidad. 


\section{Conclusiones}

¿Es la Universidad de Sevilla una institución que abre o cierra puertas a los estudiantes con discapacidad? Este estudio nos ha permitido conocer la relación de este grupo de estudiantes con la propia institución, identificando aquellos aspectos que consideran ayudas y las principales barreras a las que se enfrentan en el momento de su acceso y permanencia en las Universidad.

Del análisis realizado en el apartado anterior pueden extraerse varias conclusiones. La primera idea importante a destacar es que han surgido numerosas coincidencias entre los estudiantes, independientemente del tipo de discapacidad y estudios cursados, tanto a la hora de identificar ayudas, como barreras. Ahora bien, en cualquier caso, el número de barreras identificadas ha sido superior al de ayudas. Es decir, los participantes reconocen que, en ocasiones, han recibido ayudas que les han facilitado su inclusión, sin embargo, ponen de manifiesto muchos más obstáculos que les suponen impedimentos día a día para su proceso de aprendizaje. Estos resultados coinciden con trabajos como los de Borland y James (1999), Castellana y Sala (2005), Hadjikakou y Hartas (2008), Prowse (2009), Ryan y Struhs, 2004 o Tinklin y Hall (1999).

Respecto a las barreras que se han identificado a nivel institucional, algunos estudiantes denuncian que la universidad debería de dotar de una mayor información al personal de la institución para atender a la diversidad. El acceso a la universidad se percibe como un momento complejo, caracterizado por esta falta de información. La dependencia de la buena voluntad del personal de la universidad - como también ha aparecido en otros trabajos internacionales, como en Fuller et al., (2004), Leyser et al. (2000), Tinklin y Hall (1999) - no tendría que ser la medida subsidiaria que alentara al estudiantes en sus trayectorias formativas. En este sentido, convendría que la universidad pudiera establecer durante el primer año académico de los estudiantes algún mecanismo de ayuda o sobre la difusión de información que posibilite a todos sus estudiantes contar desde un primer momento con orientaciones sobre la institución y su funcionamiento.

Uno de los problemas que más preocupa, al igual que ya apuntaron Fuller et al., (2004) o Moswella y Mukhopadhyay (2011) es el desconocimiento que la comunidad universitaria tiene de la normativa que regula los derechos de los estudiantes con discapacidad. En particular, la desinformación del profesorado sobre la propia existencia de estos estudiantes, así como de sus necesidades y derechos recogidos en la normativa, es una barrera que podría eliminarse de forma sencilla facilitando, por parte de la universidad al comienzo del curso, la documentación adecuada a cada profesor y, como expresa Bessant (2009), conociendo el profesorado sus obligaciones legales. 
La incertidumbre que produce el exceso de burocracia referida a las convocatorias oficiales de ayudas económicas y de movilidad es otra de las barreras comúnmente reseñadas. Una mayor agilidad en la gestión administrativa o la simplificación del procedimiento para su rápida resolución supondría que algunos estudiantes pudieran cubrir de inmediato sus necesidades al beneficiarse de estas ayudas con mayor prontitud.

Por otro lado, a pesar de que ha sido un tema muy estudiado y denunciado en los últimos años, las barreras arquitectónicas y de infraestructura siguen siendo una de los obstáculos más ampliamente señalados por estos alumnos, especialmente las que se identifican en las aulas universitarias, obsoletas en muchos casos. Es preciso reconocer que, aunque se observan avances en la respuesta que la universidad ofrece al alumnado con discapacidad, es necesario dar pasos firmes que realmente garanticen el diseño de espacios universitarios más inclusivos.

Todo lo anterior perfila algunas de las condiciones que debiera cumplir la Universidad de Sevilla para que fuera más proactiva, a saber: la información ofrecida por ésta debería ser más accesible y unificada, los distintos trámites a realizar deberían facilitarse, la normativa debería dar respuesta a sus necesidades y ser aplicada, y las aulas y otros espacios deberían ser accesibles para todos y todas.

En cuanto a las ayudas que se han identificado en este estudio, también existe bastante acuerdo a la hora de señalar la gratuidad de la matrícula como algo muy positivo de cara a sus estudios. Además, uno de los servicios mejor valorados por los participantes del estudio está relacionado con la inmediatez y continuidad en la información que reciben sobre las ayudas, becas, ofertas de trabajo y cursos de formación.

La gran mayoría de los participantes en el estudio coinciden en señalar al Servicio de Asistencia a la Comunidad Universitaria (SACU) como la principal ayuda que obtienen de la institución. No obstante, y sin perjuicio de lo anterior, este servicio debería dotarse con más medios humanos y materiales, pues la escasez de los mismos resulta una merma para su eficacia y agilidad. De igual modo, se recomienda que tanto la accesibilidad como la descentralización del servicio sean dos aspectos a considerar.

En definitiva, el reconocimiento de todas estas barreras y el fuerte compromiso por superarlas, permitirá concretar propuestas de mejora, a través de políticas, estrategias, procesos y programas, encaminadas hacia nuevas prácticas de inclusión que contribuyan a que la universidad sea también un espacio de excelencia en lo que se refiere a la educación inclusiva, participativa y en igualdad de oportunidades y condiciones. 


\section{Referencias}

ADAMS, M.; HOLLAND, S. Improving Access to higher education for disabled people. In: ADAMS, M.; BROWN, S. (Eds.). Towards inclusive learning in higher education. London: Routledge, 2006a. p.10-22.

ADAMS, M.; HOLLAND, S. Conclusion: a manifesto for mainstreaming inclusive practice. In: ADAMS, M.; BROWN, S. (Eds.). Towards inclusive learning in higher education. London: Routledge, 2006b. p.187-189.

AINSCOW, M.; MILES, S. Desarrollando sistemas de educación inclusiva: ¿cómo podemos hacer progresar las políticas? In: GINÉ, C. et al. (Coords.). La educación inclusiva. De la exclusión a la plena participación de todo el alumnado. Barcelona: ICE-Horsori, 2009. p.161-170.

ARMSTRONG, F.; BARTON, L. Is there anyone there concerned with human rights? Cross-cultural connections, disability and the struggle for change in England. In: ARMSTRONG, F.; BARTON, L. (Eds.). Disability, human rights and education: Cross cultural perspectives. Milton Keynes, UK: Open University Press, 1999. p.210-229.

BARNES, C. Disability, higher education and the inclusive society. British Journal of Sociology of Education, v.28, n.1, p.135-145, 2007.

BARON, S.; PHILLIPS, R.; STALKER, K. Barriers to training for disabled social workstudents. Disability and Society, n.11, p.361-377, 1996.

BARTON, L. Markets, managerialism and inclusive education. In: CLOUGH, P. (Ed.). Managing inclusive education: From policy to experience. London: Paul Chapman, 1998. p.78-91.

BAUSELA HERRERAS, E. Atención a la diversidad en educación superior. Profesorado, revista de curriculum y formación del profesorado, n.6, p.1-2, 2002. Recuperado de: http://www.ugr. es/ $\sim$ recfpro/rev61COL4.pdf. Acesso em: set. 2014.

BEAUCHAMP-PRYOR, K. From absent to active voices: securing disability equality within higher education. International Journal of Inclusive Education, v.16, n.3, p. 283-295, 2012.

BESSANT, J. Measuring up? Students, disability and assessment in the university. Assessment in different dimensions. A conference on teaching and learning in Terciary Education. Melbourne, 2009. p.28-40.

BIKLEN, D. Constructing inclusion: lessons from critical, disability narratives. International Journal of Inclusive Education, v.4, n.4, p.337- 353, 2000.

BORLAND, J.; JAMES, S. The learning experience of students with disabilities in higher education. A case study of a UK university. Disability \& Society, v.14, n.1, p.85-101, 1999.

BOXALL, K., CARSON, I.; DOCHERTY, D. Room at the academy? People with learning difficulties and higher education. Disability \& Society, v.19, n.2, p.99-112, 2004.

CASTELLANA, M.; SALA, I. Estudiantes con discapacidad en aulas universitarias. (Students with disabilities in university classrooms). Comunicación presentada en el I Congreso Nacional de Universidad y Discapacidad, Salamanca (Paper presented at the 1st National Convention University and Disability, in Salamanca), nov. 2005.

CLAIBORNE, L. et al. Supporting students with impairments in higher education: social inclusion or cold comfort? International Journal of Inclusive Education, v.15, n.5, p.513-527, 2010.

CORBETT, J.; SLEE, R. An international conversation on inclusive education. In: ARMSTRONG, F.; ARMSTRONG, D.; BARTON, L. (Eds.). Inclusive education: policy, contexts and comparative perspective. London: David Fulton, 2000. p. 133-146.

DÍAZ SÁNCHES, F.A. Importancia de la orientación educativa en la atención a la diversidad de alumnoslas con necesidades educativas especiales. Narración de experiencias. In: SALMERON, V.; LÓPEZ, V. L. (Coords.). Orientación Educativa en las Universidades. Granda: Grupo Editorial Universitario, 2000. p.269-272.

FERNI, T.; HENNING, M. From a disabling world to a new vision. In: ADAMS, M.; BROWN, S. (Eds.). Towards inclusive learning in higher education. London: Routledge, 2006. p.23-31. 
FOREMAN, P. et al. Characteristics, Academic and Post-University Outcomes of Students with a Disability at the University of Newcastle. Higher Education Research \& Development, v.20, n.3, p.313-325, 2001.

FRANK, A. W. Practicing Dialogical Narrative Analysis. In: HOLSTEIN, J. A. ; GUBRIUM, J. F. (Eds.). Varieties of Narrative Analysis. Los Angeles, CA: Sage Publications, 2011. p.33-52.

FULLER, M., BRADLEY, A.; HEALEY, M. Incorporating disabled students within an inclusive higher education environment. Disability \& Society, v.19, n.5, p.455-468, 2004.

FULLER, M. et al. Barriers to learning: a systematic study of the experience of disabled students in one university. Studies in Higher Education, v.29, n.3, p.303-318, 2004.

FULLER, M. et al. What are disabled students' experiences of learning at university? Social Diversity and Difference. 2005. Recuperado de: http://arrts.gtcni.org.uk/gtcni/ bitstream/2428/49115/2/keele.docds.pdf. Acesso em: set. 2014.

GOODLEY, D. et al. Researching life stories. London: Routledge, 2004.

HADJIKAKOU, K.; HARTAS, D. Higher education provision for students with disabilities in Cyprus. Higher Education, n.55, p.103-119, 2008.

HOLLOWAY, S. The experience of higher education from the perspective of disabled students. Disability \& Society, v.16, n.4, p.597-615, 2001.

HOPKINS, L. The path of least resistance: a voice-relational analysis of disabled students' experiences of discrimination in English universities. International Journal of Inclusive Education, v.15, n.7, p.711-727, 2011.

HURST, A. Reflecting on researching disabilities and higher education, in: BARTON, L. (Ed.). Disabilities and Society: emerging issues and insights. London: Longman, 1996. p.123-143.

JACKLIN, A. et al. Improving the experiences of disabled students in higher education. 2007. Recuperado de: http://www.heacademy.ac.uk/assets/documents/research/jacklin.pdf. Acesso em: 4 set. 2014.

KONUR, O. Assessment of Disabled Students in Higher Education: Current public policy issues. Assessment and Evaluation in Higher Education, v.27, n.2, p.131-152, 2002.

KONUR, O. Teaching disabled students in higher education. Teaching in Higher Education, n.11, p.351- 363, 2006.

LEYSER, Y. et al. Students with disabilities in higher education: perspectives of American and Israeli faculty members. International Education, n.29, p.47-67, 2000.

MATTHEWS, N. Teaching the "invisible" disabled students in the classroom: disclosure, inclusion and the social model of disability. Teaching in Higher Education, v.14, n.3, p.229239, 2009.

MCCLUSKEY, G. Exclusion from school: what can "included" pupils tell us? British Educational Research Journal, v.34, n.4, p.447-466, 2008.

MESSIOU, K. Conversations with children: making sense of marginalization in primary school setting. European Journal of Special Need Education, v.21, n.1, p.39-54, 2006.

MILES, M. B.; HUBERMAN, A. M. Qualitative data analysis. Beverly Hills, CA: Sage Publications, 1994.

MORENO, M. T. Informe sobre la educación en América Latina y el Caribe. Documento presentado en Seminario Regional sobre la Integración de las Personas con Discapacidad en ta Educación Superior en América Latina y el Caribe. Caracas: Iesalc/Unesco, 2005.

MORIÑA, A.; CORTÉS, M. D.; MELERO, N. Inclusive curricula in Spanish higher education? Students with disabilities speak out. Disability \& Society, n.29, p.44-57, 2014.

MOSWELA, E.; MUKHOPADHYAY, S. Asking for too much? The voices of students with disabilities in Botswana. Disability \& Society, v.26, n.3, p.307-319, 2011. 
MUNN, P.; LLOYD, G. Exclusion and excluded pupils. British Educational Research Journal, v.31, n.2, p.205-221, 2005.

NIELSEN, J. A. Successful University Students with Learning Disabilities. Journal of College Student Psychotherapy, v.15, n.4, p.37-48, 2001.

NORWICH, B.; KELLY, N. Pupils' views on inclusion: moderate learning difficulties and bullying in mainstream and special schools. British Educational Research Journal, v.30, n.1, p.43-65, 2004.

OLIVER, M. The politics of disablement. Basingstoke: Macmillan, 1990.

OLIVER, M.; BARNES, C. Disability studies, disabled people and the struggle for inclusion. British Journal of Sociology of Education, v.31, n.5, p.547-560, 2010.

OLNEY, M. F.; BROCKELMAN, K. F. Out of the Disability Closet: Strategic use of perception management by select university students with disabilities. Disability \& Society, v.18, n.1, p.35-50, 2003.

ONOFRE, C. M. A Educação Inclusiva: buscando caminhos entre limites e possibilidades na Universidade. Rev. Humanidades, Fortaleza, v.21, n.1, p.11-15, 2006.

POWELL, S. Special teaching in higher education. In: POWELL, S. (Ed.). Special teaching in higher education: Successful strategies for access and inclusion. London: Kogan Page, 2003. p.3-16.

PROWSE, S. Institutional construction of disabled students. Journal of Higher Education Policy and Management, v.31, n.1, p.89-96, 2009.

RIDDELL, S. et al. Managerialism and equalities: tensions within widening access policy and practice for disabled students in UK universities. Higher Education, n.54, p.615-628, 2007.

RIDDELL, S.; WILSON, A.; TINKLIN, T. Disability and the wider access agenda: supporting disabled students in different institutional contexts. Widening Participation and Lifelong Learning, n.4, p.12-26, 2002.

RIESSMAN, C. K. Narrative methods for the human sciences. Los Angeles: Sage Publications, 2008.

RILEY, K.; DOCKING, J. Voices of disaffected pupils: implications for policy and practice. British Journal of Educational Studies, v.52, n.2, p.166-179, 2004.

RUDDUCK, J. Pupils' experiences of teaching and learning. Swindon: Economic and Social Research Council, 1996.

RYAN, J.; STRUHS, J. University education for all? Barriers to full inclusion of students with disabilities in Australian universities. International Journal of Inclusive Education, v.8, n.1, p.73-90, 2004.

SHEVLIN, M.; KENNY, M.; MCNEELA, E. Participation in higher education for students with disabilities: an Irish perspective'. Disability \& Society, v.19, n.1, p.15-30, 2004.

STEVENS, P. Pupils' perspectives on racism and differential treatment by teachers: on stragglers, the ill and being deviant. British Educational Research Journal, v.35, n.3, p.413-430, 2009.

TANGEN, R. Listening to children's voices in educational research: some theoretical and methological problems. European Journal of Special Needs Education, v.23, n.2, p.157-166, 2008.

TINKLIN, T.; HALL, J. Getting round obstacles: disabled students' experiences in higher education in Scotland. Studies in Higher Education, n.24, p.183-194, 1999.

TINKLIN, T.; RIDDELL, S.; WILSON, A. Policy and provision for disabled students in higher education in Scotland and England: The current state of play. Studies in Higher Education, v.29, n.5, p.637-657, 2004.

TOMLINSON, J. The Tomlinson report-inclusive learning. Report of the Learning Difficulties and/or Disabilities Committee. Further Education Funding Council Learning Difficulties and/or Disabilities Committee (FEFCE). 1996.

WEHMAN, P. Life beyond the classroom: Transition strategies for young people with disabilities. 4. ed. Baltimore, MD: Paul Brookes, 2006. 


\section{Resumen}

\section{¿Educación inclusiva en la Enseñanza Superior?: el caso del alumnado con discapacidad}

Este artículo presenta los resultados de una investigación (Barreras y ayudas que los estudiantes con discapacidad identifican en la Universidad, Ministerio de Ciencia e Innovación de España, ref. EDU 2010-16264) en curso que está siendo desarrollada en la Universidad de Sevilla. El propósito de ésta es analizar desde la perspectiva del alumnado con discapacidad cuáles son las barreras y ayudas que encuentran en la Enseñanza Superior (ES). Para realizar el estudio se ha hecho uso del método biográfico-narrativo, que enfatiza la importancia de que las personas hablen de ellas mismas, sin silenciar su subjetividad. La muestra del estudio está constituida por 44 estudiantes. Para el tratamiento de los datos se ha realizado un doble análisis. Para la elaboración de cada historia de vida se ha llevado a cabo un análisis narrativo y para el análisis comparativo de la información recogida con todas las técnicas y personas participantes se ha realizado un análisis estructural, con un sistema de categorías y códigos. En los resultados de este trabajo se presenta la información referida a los facilitadores y obstáculos que los estudiantes con discapacidad identifican en la Universidad de Sevilla, específicamente aquellos que hacen referencia a la Universidad como Institución - personal, espacios, normativa etc. - y al Servicio de Atención a Estudiantes con Discapacidad. Por último, en las conclusiones se retomarán las principales ideas analizadas que se discutirán con otros trabajos previos.

Palabras Clave: Enseñanza Superior. Educación Inclusiva. Educación Especial. Barreras. Ayudas.

\section{Abstract}

\section{Inclusive Education in Higher Education?: The Case of Students with Disabilities}

This article presents the results of an ongoing research (Barriers and help that students with disabilities identify at the University, Spain's Ministry of Science and Innovation, reference EDU 2010-16264) being developed at the University of Seville (Spain). The objective of this work is to analyze the barriers and help found in Higher Education from the standpoint of students with disabilities. To carry out this study, the authors began with a biographic-narrative method, which emphasizes the importance of individuals speaking about themselves, without silencing their subjectivity. The study sample included 44 students. For data analysis, a structural analysis was used, based on a system of categories and codes. The results of this work present information referring to the facilities and obstacles that students with disabilities identify at the University, specifically those regarding the university as an institution - staff, spaces, rules etc. - and the Service for Students with Disabilities. Lastly, the conclusions go back to the main ideas analyzed to discuss previous works.

Keywords: Higher Education. Inclusive Education. Special Education Barriers. Aid. 